\title{
Arterial Wall Imaging in Symptomatic Carotid Stenosis: Delayed Enhancement on MDCT Angiography
}

\author{
Su Min Ha, MD', Sang-il Suh, MD', Woo-Keun Seo, MD², Hae Young Seol, MD'
}

Objective: To evaluate progressive enhancement in the carotid arterial wall overlying plaque in the symptomatic side for patients with cerebrovascular symptoms until delayed phase using MDCTA.

Materials and Methods: Twenty-one patients (all men; ages, 49-82 years; mean, $67.8 \pm 8.4$ years) with recent stroke and severe extracranial carotid stenosis were retrospectively analyzed. Pre-, early- and delayed phase images of MDCTA were obtained, and Hounsfield units (HU) of carotid walls were measured. We also measured HU of the asymptomatic contralateral carotid arterial wall for comparison. Friedman's test and Wilcoxon signed-rank test were used to evaluate the differences between groups.

Results: The averaged HU of the carotid wall in the symptomatic side was higher on the delayed phase $(65.8 \pm 14.2 \mathrm{HU})$ compared to early arterial phase $(54.2 \pm 12.6 \mathrm{HU})$. The averaged HU difference of wall enhancement between pre-contrast and delayed phase $(28.0 \pm 14.8 \mathrm{HU})$ was significantly higher than the between pre-contrast and early arterial phase $(16.4 \pm 12.1 \mathrm{HU})$ with $\mathrm{P}$ $<0.05$. In analysis of the contralateral asymptomatic side, the HU difference between pre-contrast and delayed phase $(15.5 \pm 12.0 \mathrm{HU})$ showed no significant higher value than between pre-contrast and early arterial phase $(14.9 \pm 10.9 \mathrm{HU})$.

Conclusion: The pronounced enhancement of the carotid wall in the delayed phase on MDCTA was demonstrated in symptomatic patients with severe internal carotid artery stenosis. In the future, we need more comparative studies to verify this finding as one of risk stratification.

Key Words: Carotid plaque; Delayed phase; Computed tomography angiography; Stability

'Department of Radiology, Korea University Guro Hospital, Korea University College of Medicine, Seoul, Korea 'Department of Neurology, Korea University Guro Hospital, Korea University College of Medicine, Seoul, Korea Received January 21, 2016; accepted after revision January 25, 2016.

Correspondence to: Sang-il Suh, MD, PhD, Department of Radiology, Korea University Guro Hospital, Korea University College of Medicine, 97 Guro-dong, Guro-gu, Seoul 152-703, Korea.

Tel. 82.2.2626.3215 Fax. 82.2.863.9282 E-mail: sangil.suh@gmail.com

This is an Open Access article distributed under the terms of the Creative Commons Attribution Non-Commercial License (http://creativecommons.org/licenses/by-nc/3.0) which permits unrestricted non-commercial use, distribution, and reproduction in any medium, provided the original work is properly cited. 
Extracranial carotid stenosis accounts for $15-20 \%$ of all ischemic stroke, and the degree of luminal stenosis has been considered an important risk factor for stroke and a standard parameter for stenosis severity caused by atherosclerosis, according to the North American Symptomatic Carotid Endarterectomy Trial (NASCET) [1]. However, in recent years, there has been more emphasis on morphology and composition of plaque, giving rise to the concept of vulnerable plaque $[2,3]$.

Noninvasive image modalities, such as ultrasound, positron emission tomography (PET), magnetic resonance imaging (MRI), and multi-detector-CT angiography (MDCTA), have demonstrated that certain morphological features of vulnerable plaque, such as a large lipid-rich necrotic core, intraplaque hemorrhage, neovascularization, inflammation, or thin fibrous caps, are associated with risk of stroke [4]. Among them, hyperplasia of the adventitial vasa vasorum and neovascularization are important features of plaque development, and association between neovascularization and plaque vulnerability has been suggested [5-8]. Some authors have indicated that carotid wall enhancement in the delayed phase on dynamic contrast-enhanced MRI is a reliable sign of plaque neovascularization and inflammation linked to increased risk of clinical vascular events [9-11]. Recently, Romero et al. [12] demonstrated early arterial CTA enhancement of the ICA wall is significantly more common in symptomatic than in asymptomatic patients with more than 70\% ICA stenosis.

The purpose of this preliminary study is to evaluate progressive contrast enhancement carotid artery wall overlying plaque of the symptomatic side in patients with cerebrovascular symptoms until the delayed phase using MDCTA and assess whether there is a statistical association between increase in Hounsfield Unit (HU) of the carotid wall in the delayed phase and cerebrovascular symptoms.

\section{MATERIALS AND METHODS}

\section{Patient population}

Clinical and imaging data, obtained as part of the standard clinical stroke care at our institution, were retrospectively reviewed with approval of the institutional review board. Between April 2009 and November 2011, 21 patients (all men; ages, 49-82 years; mean, $67.8 \pm 8.4$ years) with $>70 \%$ carotid stenosis on MDCTA (according to NASCET) [13] within 2 weeks from ischemic episode and in whom carotid stent was inserted were enrolled in this retrospective study. The patients were defined as "symptomatic" based on clinical presentation at admission and positive diffusion-weighted imaging at follow-up during admission. We defined the "asymptomatic" side as the contralateral carotid artery with no relevant correlation to clinical symptoms in a patient.

\section{MDCTA protocol}

All patients were scanned from the aortic arch to the head using a 64-multi-detector-row CT scanner (Brilliance 64, Philips Medical Systems, Best, The Netherlands) with a standardized optimized contrastenhanced CT angiography protocol.

In our protocol for the analysis of carotid arteries, an unenhanced baseline acquisition of the entire carotid artery was performed. An automatic bolus-tracking program was used to start acquisition after contrast injection (370 mg I/ml, Ultravist 370, Bayer Schering Pharma AG, Berlin, Germany). After the start of contrast material injection, the software measured the attenuation value for a region of interest (ROI) within the ascending aorta and scanning started after 3.2 seconds as soon as the threshold of $150 \mathrm{HU}$ was exceeded. The injection rate of contrast media was 6 $\mathrm{ml} / \mathrm{sec}$, for a total volume of $100 \mathrm{ml}$. CT technical parameters included: $120 \mathrm{mAs}, 120 \mathrm{kV}$, a $1-\mathrm{mm}$ slice thickness, $64 \times 0.625 \mathrm{~mm}$ detector configuration using a pitch of 0.89 for pre-contrast phase; $180 \mathrm{mAs}$, $120 \mathrm{kV}$, a pitch of 0.89 for early arterial phase; 100 mAs, $120 \mathrm{kV}$, a pitch of 0.67 for delayed phase $(90 \mathrm{sec}$ after contrast injection). Subsequently, the images were processed at a workstation to create multiplanar reconstruction for analysis of degree of stenosis and plaque morphology. The total effective radiation dose during study averaged about $4.02 \pm 0.2 \mathrm{mSv}$.

\section{Image analysis}

We evaluated severity of stenosis according to the NASCET and carotid plaque wall on pre-contrast, early arterial, and delayed phase measured in Hounsfield units (HU). Carotid wall enhancement was considered present if $>50 \%$ of the carotid wall circumference was enhanced at the level of maximum stenosis with a 10HU increase.

Bilateral carotid vessels were evaluated for each patient to comparatively evaluate the asymptomatic side. The MDCTA were separately analyzed by two experienced neuroradiologists by consensus. In the contrast-enhanced early and delayed phases, a circular ROI, three to five in number and with $1 \mathrm{~mm}$ in diameter, in the predominant enhancing area of carotid 
wall overlying plaque was used to measure $\mathrm{HU}$ value at the most stenotic site (Fig. 1). A ROI of the same area as that used in the contrast-enhanced phase was positioned in the same position at carotid artery wall to measure the unenhanced $\mathrm{HU}$ value at an equivalent level. In order to measure the degree of enhancement, we calculated the HU difference between pre-contrast and contrast enhanced early arterial and delayed phases. During the image review, the center level was set at 100-150 HU and the window width at 250-350 $\mathrm{HU}$, which allowed for optimal plaque component distinction. Severe calcified components covering the vessel wall were excluded from the ROI.

\section{Statistical analysis}

For the analysis of the data, averaged HU values between unenhanced and early arterial and delayed phase were calculated. Friedman's test was used to correlate averaged HU values and the difference of the carotid wall between pre-contrast and contrastenhanced phases within each symptomatic and asymptomatic group. The Wilcoxon signed-rank correlation test was used to test averaged HU values and difference between symptomatic and asymptomatic groups. Differences were defined as significant at a probability level $<0.05$. All analyses were performed using the SPSS 15.0 statistical package for Windows (SPSS Inc., Chicago, IL, USA).

\section{RESULTS}

The clinical characteristics of the study population are as follows: hypertension in 19 patients $(90.5 \%)$; diabetes mellitus in 7 (33.3\%); hyperlipidemia in 11
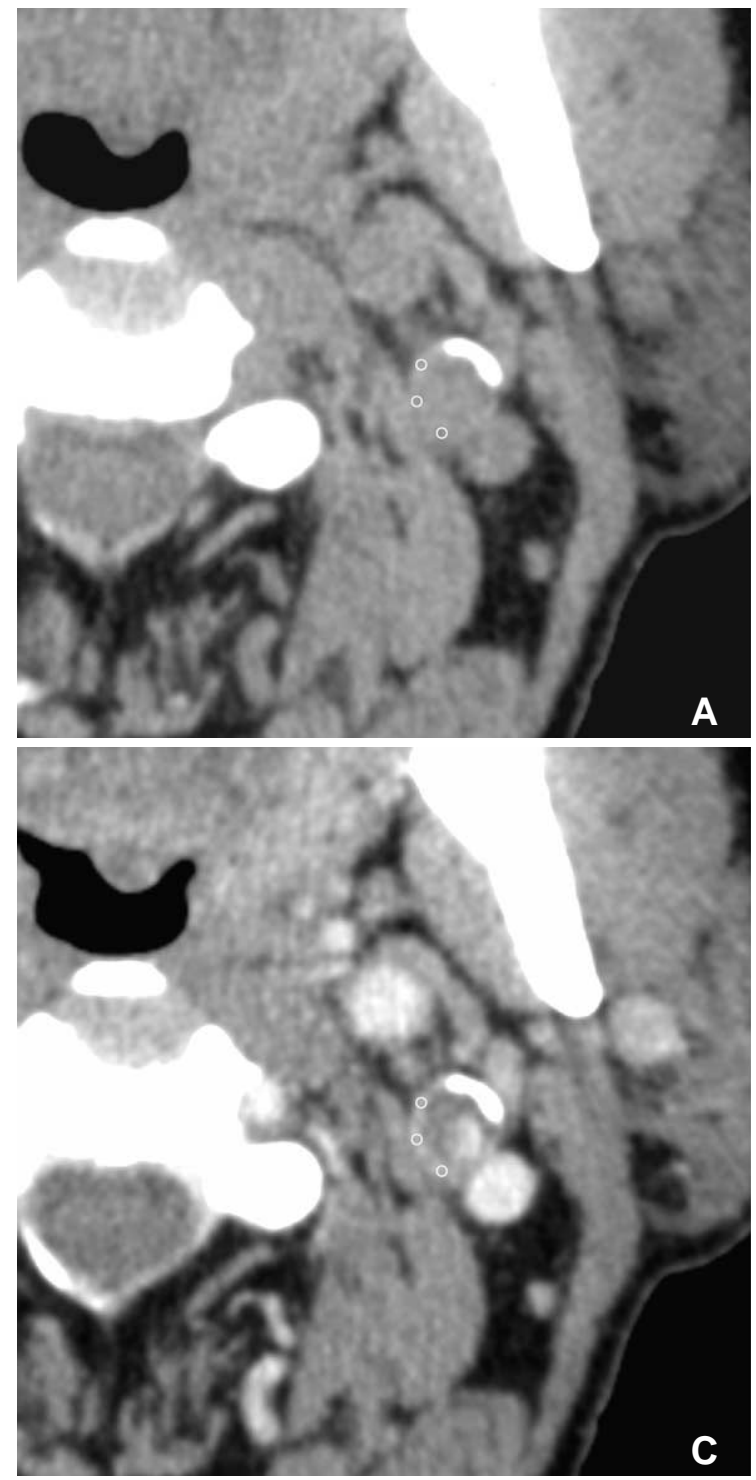

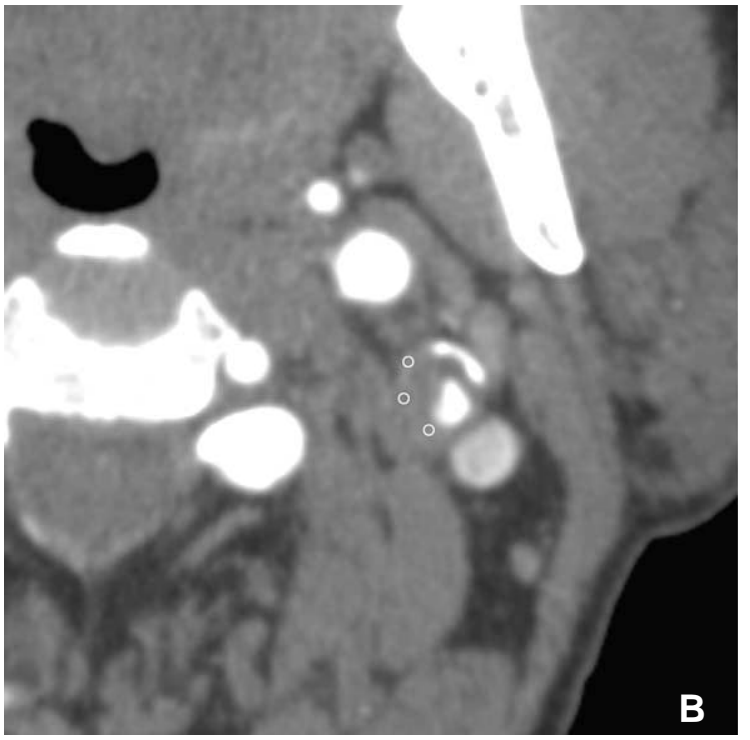

Fig. 1. A 75-year-old symptomatic male patient with acute infarctions at the left frontal and parietal lobes. MDCT axial images in the before (A), early arterial (B), and delayed (90 sec) phases (C) after administration of contrast material. The circles indicate the region of interest in the wall of the left proximal internal carotid artery (ICA). The mean $\mathrm{HU}$ of wall in each phase measured $58.6 \mathrm{HU}$, $73.6 \mathrm{HU}$, and $92 \mathrm{HU}$, respectively. The $\mathrm{HU}$ difference between the delayed $(90 \mathrm{sec})$ and pre-contrast (A) phases was 33.4, while differences between early arterial $(\mathbf{B})$ and pre-contrast $(\mathbf{A})$ phases measured 15. 
$(52.4 \%)$. The mean luminal stenosis degree in the symptomatic side measured $82 \%$ (range, $70-99 \%$ ), and the mean luminal stenosis degree in the contralateral asymptomatic side measured 40\% (range, 0-99\%).

The HU of carotid wall enhancement overlying plaque and statistical analysis in all patients are demonstrated in Table 1 . In the symptomatic side of all patients, the mean HU of carotid wall enhancement at the stenotic portion was higher in the delayed phase $(65.8 \pm 14.2 \mathrm{HU})$ compared to the early arterial phase $(54.2 \pm 12.6 \mathrm{HU})$. The HU difference of carotid wall enhancement between the pre-contrast and delayed phase $(28.0 \pm 14.8 \mathrm{HU})$ was significantly higher than between the pre-contrast and early arterial phase (16.4 $\pm 12.1 \mathrm{HU}$ ) with $\mathrm{P}<0.05$. In contrast, in the asymptomatic contralateral side, the averaged HU difference between the pre-contrast and delayed phase (15.5 \pm 12.0 HU) showed no significance higher than between the pre-contrast and early arterial phase $(14.9 \pm 10.9$ $\mathrm{HU}$ ) with $\mathrm{P}=0.05$.

Between symptomatic and asymptomatic sides, relative statistically significance existed in the averaged $\mathrm{HU}$ value and difference of pre-contrast and delayed phase $(28.0 \pm 14.8 \mathrm{HU}$ vs. $15.5 \pm 12.0 \mathrm{HU}, \mathrm{P}=$ $0.008)$. However, there was no significant correlation in the averaged $\mathrm{HU}$ value and the difference between the pre-contrast and early arterial phase between the symptomatic $(16.4 \pm 12.1 \mathrm{HU})$ and asymptomatic side $(14.9 \pm 10.9 \mathrm{HU})$ with $\mathrm{P}=0.823$.

\section{DISCUSSION}

For all patients in this study, there was a statistically higher HU difference of carotid wall overlying plaque between the pre-contrast and delayed phases than the HU difference between the pre-contrast and early arterial phases demonstrated on the symptomatic side (Fig. 2). This carotid wall enhancement that we observed may be attributed to neovascularization and inflammation. Plaque neovascularization, first described by Koester is thought to participate intimately in the growth and progression of human atherosclerosis [14]. It has previously been demonstrated, by using different imaging techniques, that enhancement of carotid plaque correlated with increase of neovessels within the carotid plaque and that the cause of enhancement is due to increased vascularity of the adventitial vasa vasorum feeding the plaque neovasculature [15-17]. Kerwin et al. [11] reported that, on dynamic contrast-enhanced MRI, temporal increase in enhancement on the outer rim or adventitia until delayed phase due to neovascularization is a sign of plaque inflammation and vulnerability linked to increased risk of clinical vascular events. Staub et al. [18] confirmed pronounced enhancement of the adventitial vasa vasorum on contrast-enhanced carotid ultrasound was associated with clinical events and that the presence of vasa vasorum-derived intraplaque neovascularization was associated with plaque instability. Romero et al. [12] recently reported CTA enhancement of the ICA wall is significantly more common in symptomatic than in asymptomatic patients with severe stenosis and explained it as vasa vasorum noevascularization in the early arterial phase. To our knowledge, this is the first study that examined carotid wall enhancement until the delayed phase using MDCTA and its correlation with a patient's clinical history.

In contrast to our study, recently it has been reported

Table 1. Analysis of Mean CT HU in Carotid Wall Overlying Plaque on CTA Axial Images

\begin{tabular}{|c|c|c|c|}
\hline & Symptomatic side* $(n=21)$ & Asymptomatic side ${ }^{\star *}(n=21)$ & $P^{\star \star *}$ \\
\hline HU in early phase (a) & $54.2 \pm 12.6$ & $51.3 \pm 13.2$ & 0.668 \\
\hline HU in delay phase (b) & $65.8 \pm 14.2$ & $51.9 \pm 12.9$ & 0.004 \\
\hline$\Delta \mathrm{HU}$ from pre-contrast to delayed phase (c) & $28.0 \pm 14.8$ & $15.5 \pm 12.0$ & 0.008 \\
\hline$\Delta \mathrm{HU}$ from pre-contrast to early phase (d) & $16.4 \pm 12.1$ & $14.9 \pm 10.9$ & 0.823 \\
\hline
\end{tabular}

Note. - Values are mean \pm standard deviation.

CTA, computed tomography angiography; HU, Hounsfield unit

${ }^{*} \mathrm{HU}(\mathrm{b})$ is statistically higher than $\mathrm{HU}(\mathrm{a}) \& \Delta \mathrm{HU}(\mathrm{c})$ is statistically higher than $\Delta \mathrm{HU}$ (d) with $\mathrm{P}$ value $<0.001$ in symptomatic side by using Friedman test.

${ }^{* *} \mathrm{HU}(\mathrm{b})$ is statistically not higher than $\mathrm{HU}(\mathrm{a}) \& \Delta \mathrm{HU}(\mathrm{c})$ is statistically not higher than $\Delta \mathrm{HU}(\mathrm{d})$ with $\mathrm{P}$ value $>0.05$ in asymptomatic side by using Friedman test.

*** $\mathrm{P}$ values show statistically higher $\mathrm{HU}(\mathrm{b}) \& \Delta \mathrm{HU}(\mathrm{c})$ in symptomatic side than those of asymptomatic side by using Wilcoxon signed-rank correlation test. 

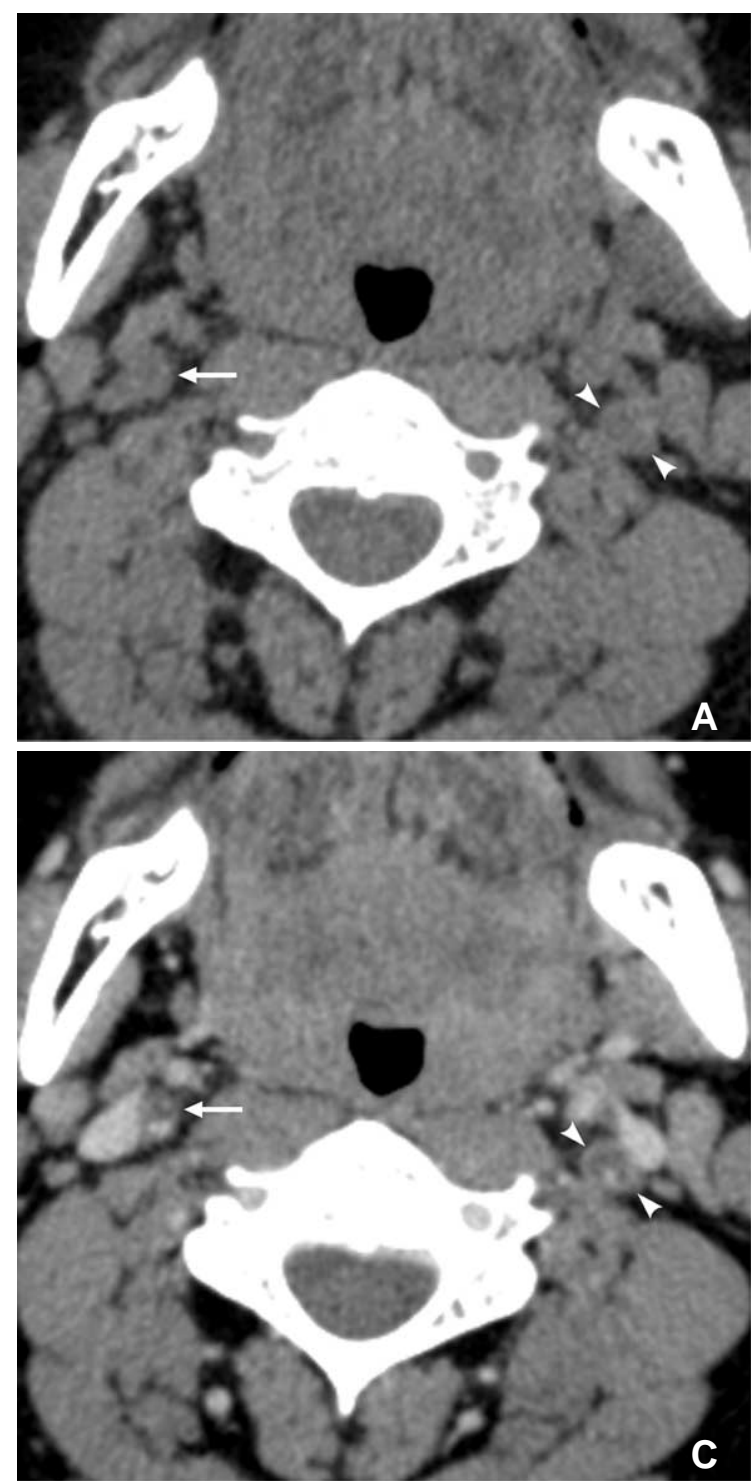

that an increase in HU from the early to delayed phases on MDCTA indicates plaque stability [19]. However, their study analyzed dynamic contrast enhancement of variable plaque components, including fibrous tissue, lipid core, intraplaque hemorrhage, and neovascularization, with free-hand segmentation of the ROI. In contrast, considering heterogeneity in the carotid plaque component, we focused on wall enhancement overlying the carotid plaque, and hypothesized that it would reflect plaque vulnerability more accurately and constantly with respect to adventitial neovascularization. This different approach may explain the different results in our study. Additionally, Saba et al. [20] recently reported that using contrast enhanced MDCTA, contrast plaque enhancement is statistically associated with neovascularization and microvessel

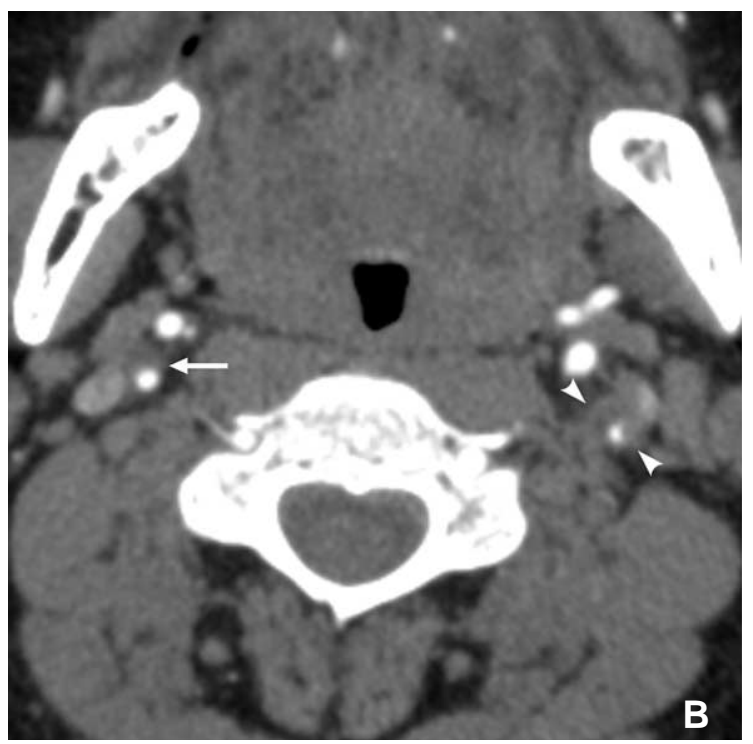

Fig. 2. A 71-year-old symptomatic male patient with acute cerebral infarction at left middle cerebral artery territory. MDCTA axial images in the before (A), early arterial (B), and delayed (90 sec) (C) phases after contrast enhancement. Compared to the asymptomatic right side (arrow), the symptomatic left carotid arterial wall overlying the plaque demonstrates more prominent and discrete enhancement in the delayed phase image (arrowheads).

density in symptomatic patients who underwent carotid endarterectomy. However, our study analyzed the arterial wall enhancement overlying plaque specifically, and our result may be complementary and allow better understanding of the behavior of vulnerable and nonvulnerable plaque.

Some limitations in this study should be addressed. First, MDCTA leads to ionizing radiation exposure [21]. However, CTA is a routine and adjunctive imaging modality that is obtained as part of the standard of care in a few seconds, without any requirement for a specific protocol in acute or chronic cerebrovascular disease. Moreover, MDCTA is a useful and noninvasive modality that gives information about the degree of luminal stenosis, extent of intra-plaque calcification, or ulceration with acceptable range of 


\section{Arterial Wall Imaging in Symptomatic Carotid Stenosis}

radiation during our study. Second, our study used contralateral carotid arteries as controls, since it has the advantage of accounting for various factors such as age and cardiovascular risk factors. However, an alternative method could have used asymptomatic age-matched controls with severe atherosclerotic disease. Third, this study did not quantify $\mathrm{HU}$ for the entire plaque volume with free-hand segmentation for a ROI because of technical difficulty and limited spatial resolution. However, we selected the circle segmentation ROI method to measure the carotid wall HU specifically, because we hypothesized this method would be more accurate in measuring the wall in which we are interested. Finally, we could not correlate findings with histologic results, because all patients with severe stenosis were eligible for intervention, which in our hospital includes carotid stenting; and unfortunately, it is not possible to obtain preserved carotid endarterectomy specimens that include an adventitial layer.

In conclusion, the pronounced enhancement of the carotid wall in the delayed phase on MDCTA was seen in symptomatic patients with severe internal carotid artery stenosis. In the future, we need more comparative studies to verify this finding as one of risk stratification.

\section{References}

1. Chaturvedi S, Bruno A, Feasby T, Holloway R, Benavente O, Cohen SN, et al. Carotid endarterectomy--an evidence-based review: report of the Therapeutics and Technology Assessment Subcommittee of the American Academy of Neurology. Neurology 2005;65:794-801

2. Ballotta E, Da Giau G, Renon L. Carotid plaque gross morphology and clinical presentation: a prospective study of 457 carotid artery specimens. J Surg Res 2000;89:78-84

3. Rothwell PM, Gibson R, Warlow CP. Interrelation between plaque surface morphology and degree of stenosis on carotid angiograms and the risk of ischemic stroke in patients with symptomatic carotid stenosis. On behalf of the European Carotid Surgery Trialists' Collaborative Group. Stroke 2000;31:615-621

4. U-King-Im JM, Young V, Gillard JH. Carotid-artery imaging in the diagnosis and management of patients at risk of stroke. Lancet Neurol 2009;8:569-580

5. Fleiner M, Kummer M, Mirlacher M, Sauter G, Cathomas G, Krapf R, et al. Arterial neovascularization and inflammation in vulnerable patients: early and late signs of symptomatic atherosclerosis. Circulation 2004;110:2843-2850

6. McCarthy MJ, Loftus IM, Thompson MM, Jones L, London NJ, Bell PR, et al. Angiogenesis and the atherosclerotic carotid plaque: an association between symptomatology and plaque morphology. J Vasc Surg 1999;30:261-268
7. Mofidi R, Crotty TB, McCarthy P, Sheehan SJ, Mehigan D, Keaveny TV. Association between plaque instability, angiogenesis and symptomatic carotid occlusive disease. Br J Surg 2001;88:945-950

8. Dunmore BJ, McCarthy MJ, Naylor AR, Brindle NP. Carotid plaque instability and ischemic symptoms are linked to immaturity of microvessels within plaques. J Vasc Surg 2007;45:155-159

9. Moulton KS, Vakili K, Zurakowski D, Soliman M, Butterfield C, Sylvin E, et al. Inhibition of plaque neovascularization reduces macrophage accumulation and progression of advanced atherosclerosis. Proc Natl Acad Sci U S A 2003;100:4736-4741

10. Celletti FL, Waugh JM, Amabile PG, Kao EY, Boroumand S, Dake MD. Inhibition of vascular endothelial growth factormediated neointima progression with angiostatin or paclitaxel. $J$ Vasc Interv Radiol 2002;13:703-707

11. Kerwin WS, O’Brien KD, Ferguson MS, Polissar N, Hatsukami TS, Yuan C. Inflammation in carotid atherosclerotic plaque: a dynamic contrast-enhanced MR imaging study. Radiology 2006;241:459-468

12. Romero JM, Babiarz LS, Forero NP, Murphy EK, Schaefer PW, Gonzalez RG, et al. Arterial wall enhancement overlying carotid plaque on CT angiography correlates with symptoms in patients with high grade stenosis. Stroke 2009;40:1894-1896

13. Wardlaw JM, Chappell FM, Best JJ, Wartolowska K, Berry E; NHS Research and Development Health Technology Assessment Carotid Stenosis Imaging Group. Non-invasive imaging compared with intra-arterial angiography in the diagnosis of symptomatic carotid stenosis: a meta-analysis. Lancet 2006;367:1503-1512

14. Moulton KS. Angiogenesis in atherosclerosis: gathering evidence beyond speculation. Curr Opin Lipidol 2006;17:548-555

15. Kerwin W, Hooker A, Spilker M, Vicini P, Ferguson M, Hatsukami T, et al. Quantitative magnetic resonance imaging analysis of neovasculature volume in carotid atherosclerotic plaque. Circulation 2003;107:851-856

16. Coli S, Magnoni M, Sangiorgi G, Marrocco-Trischitta MM, Melisurgo G, Mauriello A, et al. Contrast-enhanced ultrasound imaging of intraplaque neovascularization in carotid arteries: correlation with histology and plaque echogenicity. $\mathrm{J} \mathrm{Am} \mathrm{Coll}$ Cardiol 2008;52:223-230

17. Xiong L, Deng YB, Zhu Y, Liu YN, Bi XJ. Correlation of carotid plaque neovascularization detected by using contrast-enhanced US with clinical symptoms. Radiology 2009;251:583-589

18. Staub D, Patel MB, Tibrewala A, Ludden D, Johnson M, Espinosa $\mathrm{P}$, et al. Vasa vasorum and plaque neovascularization on contrastenhanced carotid ultrasound imaging correlates with cardiovascular disease and past cardiovascular events. Stroke 2010;41:41-47

19. Horie N, Morikawa M, Ishizaka S, Takeshita T, So G, Hayashi K, et al. Assessment of carotid plaque stability based on the dynamic enhancement pattern in plaque components with multidetector CT angiography. Stroke 2012;43:393-398

20. Saba L, Lai ML, Montisci R, Tamponi E, Sanfilippo R, Faa G, et al. Association between carotid plaque enhancement shown by multidetector CT angiography and histologically validated microvessel density. Eur Radiol 2012;22:2237-2245

21. Chalela JA. Evaluating the carotid plaque: going beyond stenosis. Cerebrovasc Dis 2009;27 Suppl 1:19-24 